\title{
A Scattering Theory for Markov Chains
}

\author{
B. KÜMMERER
}

Mathematisches Institut A

Universität Stuttgart

Pfaffenwaldring 57

D-70569 Stuttgart

Germany

kuem@mathematik.uni-stuttgart.de

H. MAASSEN

Mathematisch Instituut

Katholieke Universiteit Nijmegen

Toernooiveld 1

6526 ED Nijmegen

The Netherlands

maassen@sci.kun.nl

\begin{abstract}
:
In the operator algebraic formulation of probability theory Markov processes typically appear as perturbations of Bernoulli processes. We develop a scattering theory for this situation. This theory applies to the isomorphism problem between Markov processes and Bernoulli shifts as well as to the description of open quantum systems.
\end{abstract}




\section{Introduction.}

The need to extend classical probability theory in order to include quantum mechanical phenomena has led to an operator algebraic formulation of probability theory ([AFL], [Par], [Mey], [Küm1,3], [KüMa], [Bia]). Here stationary stochastic processes appear as groups of automorphisms of von Neumann algebras with faithful normal states. It has turned out that stationary Markov processes are typically perturbations of Bernoulli processes (Cf. the 'quantum Feynman-Kac formula' of $[\mathrm{Acc}]$ or the 'coupling representation', of [Küm2,3,4], [KüMa]). It is therefore natural to compare the Markov evolution and the Bernoulli shift, i.e., to develop a scattering theory in the spirit of $[\mathrm{LPh}]$. The aim of the present paper is to start such an investigation. We find manageable criteria for scattering operators to exist. They lead to embeddings of Markov processes into Bernoulli shifts, in good cases to conjugacy, as studied in [KeS] and [FrO]. These embeddings can be viewed as algebraic versions of moving average representations. On the physical side this scattering theory generalises the so-called 'input-output formalism' of quantum optics ([WaM]), and makes it possible to describe in stochastic terms, for example, the 'dynamical Stark effect' in two-level atoms ([RoM $]$ ).

This paper is organised as follows. Sections 1 and 2 give the necessary background. Sections 3 and 4 develop criteria for the existence of scattering operators. They are applied to various situations in Sections 5, 6 and 7 .

\section{$\S 1$ Probability spaces and stochastic processes.}

By a non-commutative probability space we shall mean a pair $(\mathcal{A}, \varphi)$ consisting of a von Neumann algebra $\mathcal{A}$ equipped with a faithful normal state $\varphi$. In the case that $\mathcal{A}$ is commutative it can be represented in the form $\mathcal{A}=L^{\infty}(\Omega, \Sigma, \mu)$ for some probability space $(\Omega, \Sigma, \mu)$, where $\varphi: \mathcal{A} \rightarrow \mathbb{C}$ sends $f \in L^{\infty}(\Omega, \Sigma, \mu)$ to its expectation $\int_{\Omega} f d \mu$. The space $(\mathcal{A}, \varphi)$ becomes a pre-Hilbert space when equipped with the inner product $\langle x, y\rangle_{\varphi}:=\varphi\left(x^{*} y\right)$; the topology on $\mathcal{A}$ induced by the norm $\|x\|_{\varphi}^{2}:=\varphi\left(x^{*} x\right)$, agrees on bounded sets of $\mathcal{A}$ with the strong operator topology. By an operator $T:(\mathcal{A}, \varphi) \rightarrow(\mathcal{B}, \psi)$ we shall always mean a completely positive linear operator $T: \mathcal{A} \rightarrow \mathcal{B}$ mapping $\mathbf{1}_{\mathcal{A}}$ to $\mathbf{1}_{\mathcal{B}}$ and respecting expectations, i.e., $\psi \circ T=\varphi$. (We note that all operators on $(\mathcal{A}, \varphi)$ are contractions in the norm $\left.\|\cdot\|_{\varphi} \cdot\right)$ In particular, an automorphism $S$ of $(\mathcal{A}, \varphi)$ is a *automorphism of $\mathcal{A}$ leaving the state $\varphi$ invariant. In the commutative case, an automorphism $S$ is induced by a measure-preserving transformation $\sigma$ of $(\Omega, \Sigma, \mu): S(f)(x)=f(\sigma x)$. An operator $P:(\mathcal{A}, \varphi) \rightarrow(\mathcal{A}, \varphi)$ satisfying $P^{2}=P$ is called a conditional expectation onto its range $P \mathcal{A}$, which is automatically a von Neumann subalgebra of $\mathcal{A}$ (cf. [Küm1]). With respect to the pre-Hilbert space structure it is an orthogonal projection.

A stochastic process with values in some probability space $(\mathcal{A}, \varphi)$ is a family of *-homomorphisms ('random variables') $i_{t}:(\mathcal{A}, \varphi) \rightarrow(\widehat{\mathcal{A}}, \widehat{\varphi}),(t \in \mathbb{T})$, of the probability space onto a larger one. Such a process is called stationary if $i_{t}=\widehat{T}_{t} \circ i_{0}$ for some group of automorphisms $\left(\widehat{T}_{t}\right)_{t \in \mathbb{T}}$ of $(\widehat{\mathcal{A}}, \widehat{\varphi})$. Writing $i:=i_{0}$ we assume in addition that $i$ admits a left inverse $P:(\widehat{\mathcal{A}}, \widehat{\varphi}) \rightarrow(\mathcal{A}, \varphi)$, i.e., $P \circ i=\mathrm{Id}$, so 
$i \circ P:(\widehat{\mathcal{A}}, \widehat{\varphi}) \rightarrow(\widehat{\mathcal{A}}, \widehat{\varphi})$ is the conditional expectation from $(\widehat{\mathcal{A}}, \widehat{\varphi})$ onto the subalgebra $i(\mathcal{A})$. We shall denote such a stationary stochastic process by the quadruple $\left(\widehat{\mathcal{A}}, \widehat{\varphi}, \widehat{T}_{t} ; i\right)$. For a more detailed exposition of the general setting we refer to $[\mathrm{AFL}]$ and [Küm3].

For a stationary process $\left(\widehat{\mathcal{A}}, \widehat{\varphi}, \widehat{T}_{t} ; i\right)$ over $(\mathcal{A}, \varphi)$ we can form for any time interval $I \subset \mathbb{T}$ the von Neumann subalgebra $\mathcal{A}_{I}$ which is generated by the algebras $\widehat{T}_{t} \circ i(\mathcal{A})$, $(t \in I)$. If the algebras are commutative, then $\mathcal{A}_{I}$ is the algebra of functions which are measurable with respect to the $\sigma$-subalgebra generated by the random variables for times $t \in I$. It follows from the above definitions that the conditional expectation $P_{I}:(\widehat{\mathcal{A}}, \widehat{\varphi}) \rightarrow \mathcal{A}_{I}$ exists $([$ Küm1]).

The stationary process $\left(\widehat{\mathcal{A}}, \widehat{\varphi}, \widehat{T}_{t} ; i\right)$ is a Markov process if for all $x \in \mathcal{A}_{[0, \infty)}$ we have $P_{(-\infty, 0]}(x)=P_{\{0\}}(x)$. If $\widehat{\mathcal{A}}$ is commutative, this reduces to the standard notion of a Markov process.

Finally, for a stationary process $\left(\widehat{\mathcal{A}}, \widehat{\varphi}, \widehat{T}_{t} ; i\right)$ over $(\mathcal{A}, \varphi)$ one can form the transition operators $T_{t}:=P \circ \widehat{T}_{t} \circ i:(\mathcal{A}, \varphi) \rightarrow(\mathcal{A}, \varphi)$. In the case of a Markov process they form a semigroup ([AFL], [Küm1]).

\section{$\S 2$. Coupling representations.}

The following class of Markov processes has been introduced in [Küm1]. It will be the typical example of a coupling representation to be defined below. Given $(\mathcal{A}, \varphi)$, any other probability space $(\mathcal{B}, \omega)$, and an automorphism $C$ of the tensor product $(\mathcal{A} \otimes \mathcal{B}, \varphi \otimes \omega)$, we construct a Markov process over $(\mathcal{A}, \varphi)$ as follows. Define $(\mathcal{C}, \psi)$ as the infinite von Neumann tensor product $\bigotimes_{\mathbb{Z}}(\mathcal{B}, \omega)$ with respect to the infinite tensor product state $\bigotimes_{\mathbb{Z}} \omega$ and $S_{0}$ as the tensor right shift on it.

Put $(\widehat{\mathcal{A}}, \widehat{\varphi}):=(\mathcal{A} \otimes \mathcal{C}, \varphi \otimes \psi)$. On $\widehat{\mathcal{A}}$ define $S:=\operatorname{Id}_{\mathcal{A}} \otimes S_{0}$ as the trivial extension of $S_{0}$ to $\widehat{\mathcal{A}}$, and $C_{1}$ as the trivial extension of $C$ to $\widehat{\mathcal{A}}$ when we identify $\mathcal{A} \otimes \mathcal{B}$ with the subalgebra of $\hat{\mathcal{A}}$ generated by $\mathcal{A} \otimes \mathbf{1}$ and the 0 -th component in $\mathbf{1} \otimes \mathcal{C}=\mathbf{1} \otimes\left(\otimes_{\mathbb{Z}} \mathcal{B}\right)$ (being the identity on all other factors in $\mathbf{1} \otimes\left(\bigotimes_{\mathbb{Z}} \mathcal{B}\right)$ ).

The above situation is conveniently described by the following picture.

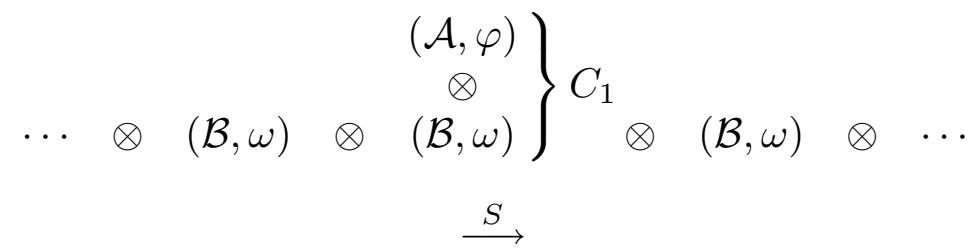

We put $\widehat{T}:=C_{1} \circ S, \widehat{T}_{n}:=\widehat{T}^{n},(n \in \mathbb{Z})$, and $i:(\mathcal{A}, \varphi) \rightarrow(\widehat{\mathcal{A}}, \widehat{\varphi}): x \mapsto x \otimes \mathbf{1}$, $P(x \otimes y):=\psi(y) \cdot x,(x \in \mathcal{A}, y \in \mathcal{C})$; then $\left(\widehat{\mathcal{A}}, \widehat{\varphi}, \widehat{T}_{t} ; i\right)$ is a Markov process ([Küm1]). It is easy to see that every classical Markov chain can be obtained in this way. In Section 5 we shall discuss an example explicitly.

The above construction can be viewed as a coupling between the probability space $(\mathcal{A}, \varphi)$ and a kind of generalised Bernoulli scheme. This is a special case of a 
representation of a Markov process by coupling to white noise, which we are now going to define.

For this definition the notion of independence is crucial. Given $(\mathcal{A}, \varphi)$ and two von Neumann subalgebras $\mathcal{A}_{1}$ and $\mathcal{A}_{2}$, we say that $\mathcal{A}_{1}$ and $\mathcal{A}_{2}$ are independent (under $\varphi)$, if $\varphi\left(x_{1} x_{2}\right)=\varphi\left(x_{1}\right) \varphi\left(x_{2}\right)$ for any elements $x_{1} \in \mathcal{A}_{1}, x_{2} \in \mathcal{A}_{2}$ and if there exist conditional expectations $P_{1}$ and $P_{2}$ onto $\mathcal{A}_{1}$ and $\mathcal{A}_{2}$. A typical example is given by $(\mathcal{A}, \varphi)=\left(\mathcal{A}_{1} \otimes \mathcal{A}_{2}, \varphi_{1} \otimes \varphi_{2}\right)$; then $\mathcal{A}_{1} \otimes \mathbf{1}$ and $\mathbf{1} \otimes \mathcal{A}_{2}$ are independent.

By $\mathcal{A} \vee \mathcal{B}$ we always denote the von Neumann algebra generated by $\mathcal{A}$ and $\mathcal{B}$.

Definition. ([Küm4]). By a (non-commutative) white noise in time $\mathbb{T}=\mathbb{Z}$ or $\mathbb{T}=\mathbb{R}$, we mean a probability space $(\mathcal{C}, \psi)$, equipped with a (weak*-continuous) automorphism group $\left(S_{t}\right)_{t \in \mathbb{T}}$ ('shift') and a filtration $\left\{\mathcal{C}_{[s, t]} \mid-\infty \leq s \leq t \leq \infty\right\}$ of von Neumann subalgebras compatible with $S_{t}$ in the sense that $S_{t}\left(\mathcal{C}_{[u, v]}\right)=$ $\mathcal{C}_{[u+t, v+t]}$ and such that

(i) $\mathcal{C}=\mathcal{C}_{\mathbb{R}}$

(ii) $\mathcal{C}_{[s, t]}=\mathcal{C}_{[s, u]} \vee \mathcal{C}_{[u, t]}$ if $-\infty \leq s \leq u \leq t \leq \infty$,

(iii) $\mathcal{C}_{[s, t]}$ and $\mathcal{C}_{[u, v]}$ are independent subalgebras of $(\mathcal{C}, \psi)$ whenever the intervals $[s, t]$ and $[u, v]$ are disjoint.

Examples of white noise come from Bernoulli schemes and their non-commutative generalisation (tensor shifts) indicated above, Gaussian white noise, and Bose/Fermi noise on the CCR/CAR algebras in quantum theory. Yet other examples exist, e.g., [Voi1,2], [BSp], [BKS].

Definition. We say that a stationary process $\left(\widehat{\mathcal{A}}, \widehat{\varphi}, \widehat{T}_{t} ; i\right)$ with values in $(\mathcal{A}, \varphi)$ has a coupling representation if the following holds. There exists a von Neumann subalgebra $\mathcal{C}$ of $\widehat{\mathcal{A}}$ and a (weak*-continuous) group of automorphisms $\left(S_{t}\right)_{t \in \mathbb{T}}$ of $(\widehat{\mathcal{A}}, \widehat{\varphi})$ such that the restriction of $S_{t}$ to $\mathcal{C}$ is a white noise with filtration $\left\{\mathcal{C}_{[s, t]} \mid-\right.$ $\infty \leq s \leq t \leq \infty\}$ such that

(i) $\widehat{\mathcal{A}}$ is generated by $i(\mathcal{A})$ and $\mathcal{C}$;

(ii) $i(\mathcal{A})$ and $\mathcal{C}$ are independent subalgebras of $\widehat{\mathcal{A}}$ under $\widehat{\varphi}$;

(iii) $\left.S_{t}\right|_{i(\mathcal{A})}$ is the identity;

(iv) For all $t \geq 0, \widehat{T}_{t}$ coincides with $S_{t}$ on $\mathcal{C}_{[0, \infty)}$ and on $\mathcal{C}_{(-\infty,-t]}$, whereas $\widehat{T}_{t}$ maps $i(\mathcal{A}) \vee \mathcal{C}_{[-t, 0]}$ onto $i(\mathcal{A}) \vee \mathcal{C}_{[0, t]}$

(v) $\mathcal{A}_{[0, t]} \subset i(\mathcal{A}) \vee \mathcal{C}_{[0, t]}$.

There is a Hilbert space analogue of this: the 'coupling structure of a unitary dilation', which has been established and studied in [KüS].

Whenever a stationary process has a coupling representation as defined above, it is a Markov process ([Küm2]). Conversely, if $\mathcal{A}=M_{n}$, Markov processes are typically of this type ([Küm2,3,4], [KüMa]).

In such a situation we define the coupling operators $C_{t}:=\widehat{T}_{t} \circ S_{-t},(t \geq 0)$. So $\widehat{T}_{t}=C_{t} \circ S_{t}$ and $\left(C_{t}\right)_{t \geq 0}$ can be extended to a cocycle of the automorphism group $S_{t}$. We consider $\left(\widehat{T}_{t}\right)_{t \in \mathbb{T}}$ as a perturbation of $\left(S_{t}\right)_{t \in \mathbb{T}}$. From our requirements we have $\left.C_{t}\right|_{\mathcal{C}_{[t, \infty)}}=\operatorname{Id}$ and $\left.C_{t}\right|_{\mathcal{C}_{(-\infty, 0]}}=$ Id for $t \geq 0$. 
There is a physical interpretation of the above coupling structure, which provides an important motivation for its study. The subalgebra $i(\mathcal{A})$ of $(\widehat{\mathcal{A}}, \widehat{\varphi})$ may be interpreted as a sytem, e.g., a radiating atom, and $\mathcal{C}$ as the surroundings (the electromagnetic field), with which it interacts. Then $S_{t}$ naturally describes the free (uncoupled) evolution of these surroundings, and $\widehat{T}_{t}$ that of the coupled system. An explicit example is described in Section 6.

\section{$\S 3 . \quad$ Scattering.}

Let us from now on assume that a Markov process $\left(\widehat{\mathcal{A}}, \widehat{\varphi}, \widehat{T}_{t} ; i\right)$ has a coupling representation to the noise $(\mathcal{C}, \psi)$ as above. We are interested in the question, under what conditions every element of $\widehat{\mathcal{A}}$ eventually ends up in the future noise algebra $\mathcal{C}_{[0, \infty)}$. In scattering theory, this property is called asymptotic completeness.

In the above physical interpretation of quantum optics this means that any observable of the atom or molecule can eventually be measured by observing the emitted radiation (cf. Section 6).

We start by defining the von Neumann subalgebra $\mathcal{A}_{\text {out }}$ of those elements in $\widehat{\mathcal{A}}$ which do eventually end up in $\mathcal{C}_{[0, \infty)}$ (Here and in the following, closures of convex sets are always taken in the $\widehat{\varphi}$-norm, equivalently in the weak* topology):

$$
\mathcal{A}_{\text {out }}:=\overline{\bigcup_{t \geq 0} \widehat{T}_{-t}\left(\mathcal{C}_{[0, \infty)}\right)} .
$$

Let $Q$ denote the conditional expectation onto the future noise algebra $\mathcal{C}_{[0, \infty)}$.

Lemma 3.1. For $x \in \widehat{\mathcal{A}}$ the following conditions are equivalent.

(a) $x \in \mathcal{A}_{\text {out }}$.

(b) $\lim _{t \rightarrow \infty}\left\|Q \widehat{T}_{t}(x)\right\|_{\widehat{\varphi}}=\|x\|_{\widehat{\varphi}}$.

(c) $\|\cdot\|_{\hat{\varphi}}-\lim _{t \rightarrow \infty} S_{-t} \circ \widehat{T}_{t}(x)$ exists and lies in $\mathcal{C}$.

The limit in (c) defines an isometric ${ }^{*}$-homomorphism $\Phi_{-}: \mathcal{A}_{\text {out }} \rightarrow \mathcal{C}$.

Proof. (a) $\Longrightarrow(\mathrm{b})$ : Choose a sequence $\left(x_{n}\right)_{n \in \mathbb{N}}$ approximating $x$ with $x_{n} \in \widehat{T}_{-n} \mathcal{C}_{[0, \infty)}$. Since $Q \widehat{T}_{n} x_{n}=\widehat{T}_{n} x_{n}$, we have

$$
\begin{aligned}
\left\|Q \widehat{T}_{n}(x)-\widehat{T}_{n}(x)\right\|_{\widehat{\varphi}} & \leq\left\|Q \widehat{T}_{n}(x)-Q \widehat{T}_{n}\left(x_{n}\right)\right\|_{\widehat{\varphi}}+\left\|\widehat{T}_{n}\left(x_{n}\right)-\widehat{T}_{n}(x)\right\|_{\widehat{\varphi}} \\
& \leq 2\left\|x-x_{n}\right\|_{\widehat{\varphi}} \longrightarrow 0 .
\end{aligned}
$$

Since $Q$ is an orthogonal projection, we have

$$
\|x\|_{\widehat{\varphi}}^{2}-\left\|Q \widehat{T}_{t}(x)\right\|_{\widehat{\varphi}}^{2}=\left\|\widehat{T}_{t}(x)\right\|_{\widehat{\varphi}}^{2}-\left\|Q \widehat{T}_{t}(x)\right\|_{\widehat{\varphi}}^{2}=\left\|\widehat{T}_{t}(x)-Q \widehat{T}_{t}(x)\right\|_{\widehat{\varphi}}^{2},
$$

so that statement (b) follows.

$(\mathrm{b}) \Longrightarrow(\mathrm{a})$ : From (b) it follows as above that $\left\|\widehat{T}_{-t} Q \widehat{T}_{t}(x)-x\right\|_{\widehat{\varphi}}^{2}=\| Q \widehat{T}_{t}(x)-$ $\widehat{T}_{t}(x)\left\|_{\widehat{\varphi}}^{2}=\right\| x\left\|_{\widehat{\varphi}}^{2}-\right\| Q \widehat{T}_{t}(x) \|_{\widehat{\varphi}}^{2} \longrightarrow 0$ as $t \longrightarrow \infty$, and hence that

$$
x=\|\cdot\|_{\widehat{\varphi}}-\lim _{t \rightarrow \infty} \widehat{T}_{-t} Q \widehat{T}_{t}(x) \in \mathcal{A}_{\text {out }} .
$$


(a) $\Longrightarrow$ (c): Let us write $\mathcal{A}_{\text {out }}^{\circ}:=\bigcup_{t>0} \widehat{T}_{-t} \mathcal{C}_{[0, \infty)}$, hence $\mathcal{A}_{\text {out }}$ is the closure of $\mathcal{A}_{\text {out }}^{\circ}$. Then for $x \in \mathcal{A}_{\text {out }}^{\circ}$, say $x \in \widehat{T}_{-u} \mathcal{C}_{[0, \infty)},(u \geq 0)$, we have, putting $t=u+s$,

$$
\lim _{t \rightarrow \infty} S_{-t} \widehat{T}_{t}(x)=\lim _{s \rightarrow \infty} S_{-u} S_{-s} \widehat{T}_{s} \widehat{T}_{u}(x)=S_{-u} \widehat{T}_{u}(x) \in S_{-u}\left(\mathcal{C}_{[0, \infty)}\right)=\mathcal{C}_{[-u, \infty)},
$$

since $\widehat{T}_{s}$ coincides with $S_{s}$ on $\mathcal{C}_{[0, \infty)}$ for $s \geq 0$. Thus an isometric *-homomorphism $\Phi_{-}: \mathcal{A}_{\text {out }}^{\circ} \rightarrow \mathcal{C}$ is defined. By an $\varepsilon / 3$-argument we prove that the limit in (c) exists for all $x \in \mathcal{A}_{\text {out }}$. As $\mathcal{C}$ is strongly closed, and on bounded sets the $\|\cdot\|_{\widehat{\varphi}}$ topology coincides with the strong operator topology, it follows that $\Phi_{-}\left(\mathcal{A}_{\text {out }}\right) \subset \mathcal{C}$.

$(\mathrm{c}) \Longrightarrow(\mathrm{a})$ : Given $x \in \widehat{\mathcal{A}}$ with $\Phi_{-}(x) \in \mathcal{C}$, choose a sequence $\left(y_{n}\right)_{n \in \mathbb{N}}$ with $y_{n} \in$ $\mathcal{C}_{[-n, \infty)}$ and $\|\cdot\|_{\widehat{\varphi}}-\lim _{n \rightarrow \infty} y_{n}=\Phi_{-}(x)$. Since $\Phi_{-}(x)=\lim _{n \rightarrow \infty} S_{-n} \widehat{T}_{n}(x)$, we have

$$
0=\lim _{n \rightarrow \infty}\left\|y_{n}-S_{-n} \widehat{T}_{n}(x)\right\|_{\widehat{\varphi}}=\lim _{n \rightarrow \infty}\left\|\widehat{T}_{-n} S_{n}\left(y_{n}\right)-x\right\|_{\widehat{\varphi}} .
$$

Since $y_{n} \in \mathcal{C}_{[-n, \infty)}$ we have $S_{n}\left(y_{n}\right) \in \mathcal{C}_{[0, \infty)}$, and $\left(\widehat{T}_{-n} S_{n}\left(y_{n}\right)\right)_{n \in \mathbb{N}}$ is a sequence in $\mathcal{A}_{\text {out }}^{\circ}$ with limit $x$.

Lemma 3.2. For all $x \in \mathcal{C}$ the limit $\|\cdot\|_{\hat{\varphi}}-\lim _{t \rightarrow \infty} \widehat{T}_{-t} \circ S_{t}(x)=: \Omega_{-}(x)$ exists and $\Phi_{-} \Omega_{-}=\operatorname{Id}_{\mathcal{C}}$. In particular, $\Phi_{-}: \mathcal{A}_{\text {out }} \rightarrow \mathcal{C}$ is an isomorphism.

Proof. Again the statement follows from the fact that for $x \in \mathcal{C}_{[-u, \infty)}$, and any $t \geq u, t=u+s, \widehat{T}_{-t} S_{t}(x)=\widehat{T}_{-u} \widehat{T}_{-s} S_{s} S_{u}(x)=\widehat{T}_{-u} S_{u}(x)$.

In scattering theory the operators $\Omega_{-}$and $\Phi_{-}$, and the related operators $\Omega_{+}:=$ $\lim _{t \rightarrow \infty} \widehat{T}_{t} \circ S_{-t}$ and $\Phi_{+}:=\lim _{t \rightarrow \infty} S_{t} \circ \widehat{T}_{-t}$ (taken as strong operator limits in the $\|\cdot\|_{\hat{\varphi}}-$ norm) are known as the Møller operators or wave operators ([LPh], [Rob]) associated to the evolutions $S_{t}$ and $\widehat{T}_{t}$.

Theorem 3.3. For a stationary process in a coupling representation the following conditions are equivalent.

(a) $\widehat{\mathcal{A}}=\mathcal{A}_{\text {out }}$.

(b) For all $x \in \mathcal{A}$ we have $\lim _{t \rightarrow \infty}\left\|Q \widehat{T}_{t} i(x)\right\|_{\hat{\varphi}}=\|x\|_{\varphi}$.

(c) The process has an outgoing translation representation, i.e. there exists an isomorphism $j:(\widehat{\mathcal{A}}, \widehat{\varphi}) \rightarrow(\mathcal{C}, \psi)$ with $\left.j\right|_{\mathcal{C}_{[0, \infty)}}=\operatorname{Id}$ such that $S_{t} \circ j=j \circ \widehat{T}_{t}$.

A coupling represention satisfying these equivalent conditions is called asymptotically complete. In probabilistic terms condition (c) is an algebraic version of the moving average representation.

Proof. The implication (a) $\Longrightarrow$ (b) follows immediately from Lemma 3.1. Conversely, suppose that (b) holds. Then by Lemma 3.1 we have that $i(\mathcal{A}) \subset \mathcal{A}_{\text {out }}$. Hence it suffices to show that

$$
\widehat{\mathcal{A}}=\overline{\bigcup_{t \in \mathbb{T}} \widehat{T}_{-t}\left(i(\mathcal{A}) \vee \mathcal{C}_{[0, \infty)}\right)} .
$$


From the properties of the coupling representation we have for $t \geq 0$

$$
\begin{aligned}
\widehat{T}_{t}\left(i(\mathcal{A}) \vee \mathcal{C}_{[-t, \infty)}\right) & =\widehat{T}_{t}\left(\left(i(\mathcal{A}) \vee \mathcal{C}_{[-t, 0]}\right) \vee \mathcal{C}_{[0, \infty)}\right) \\
& =\left(i(\mathcal{A}) \vee \mathcal{C}_{[0, t]}\right) \vee \mathcal{C}_{[t, \infty)} \\
& =i(\mathcal{A}) \vee \mathcal{C}_{[0, \infty)},
\end{aligned}
$$

and therefore

$$
\widehat{T}_{-t}\left(i(\mathcal{A}) \vee \mathcal{C}_{[0, \infty)}\right)=i(\mathcal{A}) \vee \mathcal{C}_{[-t, \infty)} .
$$

The right hand side clearly generates $\widehat{\mathcal{A}}$ as $t$ runs through $\mathbb{T}$.

(a) $\Longrightarrow$ (c): Put $j:=\Phi_{-}$, then by Lemma 3.2 we have $j(\widehat{\mathcal{A}})=\mathcal{C}$. Moreover,

$$
\begin{aligned}
S_{t} \circ j & =S_{t} \lim _{s \rightarrow \infty} S_{-s} \widehat{T}_{s} \\
& =S_{t} \lim _{u \rightarrow \infty} S_{-t} S_{-u} \widehat{T}_{u} \widehat{T}_{t} \quad \text { for } s=t+u,(u \geq 0) \\
& =\lim _{u \rightarrow \infty} S_{-u} \widehat{T}_{u} \widehat{T}_{t} \\
& =j \circ \widehat{T}_{t},
\end{aligned}
$$

and for $x \in \mathcal{C}_{[0, \infty)}$ we have

$$
j(x)=\lim _{t \rightarrow \infty} S_{-t} \widehat{T}_{t}(x)=x .
$$

(c) $\Longrightarrow($ a): Conversely, we obtain

$$
\begin{aligned}
\widehat{\mathcal{A}}=j^{-1}(\mathcal{C}) & =j^{-1}\left(\overline{\bigcup_{t \geq 0} S_{-t}\left(\mathcal{C}_{[0, \infty)}\right)}\right) \\
& =\overline{\bigcup_{t \geq 0} j^{-1} S_{-t}\left(\mathcal{C}_{[0, \infty)}\right)} \\
& =\overline{\bigcup_{t \geq 0} \widehat{T}_{-t} j^{-1}\left(\mathcal{C}_{[0, \infty)}\right)} \\
& =\bigcup_{t \geq 0} \widehat{T}_{-t}\left(\mathcal{C}_{[0, \infty)}\right) \\
& =\mathcal{A}_{\text {out }} .
\end{aligned}
$$

\section{$\S 4$. Criteria for asymptotic completeness.}

In this section we shall formulate concrete criteria for the asymptotic completeness. We restrict ourselves to the simplest case: a finite dimensional algebra $\mathcal{A}$ and a coupling of tensor type, i.e., $(\widehat{\mathcal{A}}, \widehat{\varphi})=(\mathcal{A} \otimes \mathcal{C}, \varphi \otimes \psi)$.

As before, let $Q$ denote the conditional expectation onto the future noise algebra $\mathcal{C}_{[0, \infty)}$, and let $Q^{\perp}:=\operatorname{Id}-Q$. For $t \geq 0$, let $Z_{t}$ denote the compression $Q^{\perp} \widehat{T}_{t} Q^{\perp}$ of the coupled evolution to the $\widehat{\varphi}$-orthogonal complement of the future noise. 


\section{Lemma 4.1.}

(i) $\left(Z_{t}\right)_{t \geq 0}$ is a semigroup, i.e., for all $s, t \geq 0$,

$$
Z_{s+t}=Z_{s} \circ Z_{t} .
$$

(ii) The coupling representation is asymptotically complete if and only if for all $a \in \mathcal{A}$

$$
\left\|Z_{t} i(a)\right\|_{\widehat{\varphi}} \longrightarrow 0 \quad \text { as } \quad t \longrightarrow \infty \text {. }
$$

Proof. (i). Since $\widehat{T}_{t}$ concides with $S_{t}$ on the future noise, we have $\widehat{T}_{t} \mathcal{C}_{[0, \infty)} \subset \mathcal{C}_{[0, \infty)}$ for $t \geq 0$. It follows that $\widehat{T}_{t} Q=Q \widehat{T}_{t} Q$, and therefore

$$
\begin{aligned}
Q^{\perp} \widehat{T}_{t} Q^{\perp} & =(\mathrm{Id}-Q) \widehat{T}_{t}(\mathrm{Id}-Q)=\widehat{T}_{t}-Q \widehat{T}_{t}-\widehat{T}_{t} Q+Q \widehat{T}_{t} Q \\
& =\widehat{T}_{t}-Q \widehat{T}_{t}=Q^{\perp} \widehat{T}_{t} .
\end{aligned}
$$

So, for $s, t \geq 0$,

$$
\begin{aligned}
Z_{s} Z_{t} & =Q^{\perp} \widehat{T}_{s} Q^{\perp} \widehat{T}_{t} Q^{\perp}=Q^{\perp} \widehat{T}_{s} \widehat{T}_{t} Q^{\perp} \\
& =Q^{\perp} \widehat{T}_{s+t} Q^{\perp}=Z_{s+t} .
\end{aligned}
$$

(ii). For $a \in \mathcal{A}$ we have

$$
Z_{t} i(a)=Q^{\perp} \widehat{T}_{t} Q^{\perp} i(a)=Q^{\perp} \widehat{T}_{t}(i(a)-\varphi(a) \cdot \mathbf{1})=Q^{\perp} \widehat{T}_{t} i(a),
$$

so that by Pythagoras' theorem

$$
\left\|Z_{t} i(a)\right\|_{\widehat{\varphi}}^{2}=\|a\|_{\varphi}^{2}-\left\|Q \widehat{T}_{t} i(a)\right\|_{\widehat{\varphi}}^{2} .
$$

Hence, by Theorem 3.3. the statement follows.

Theorem 4.2. Let $\left(\widehat{\mathcal{A}}, \widehat{\varphi}, \widehat{T}_{t} ; i\right)$ be a Markov process with values in a finite dimensional probability space $(\mathcal{A}, \varphi)$, which is given in a coupling representation of tensor type to a white noise. Let $Q^{\perp}$ and $Z_{t}$ be as described above, and let $e_{1}, e_{2}, \cdots, e_{n}$ be a basis of $\mathcal{A}$. Then the following conditions are equivalent.

(a) The coupling representation is asymptotically complete.

(b) for all nonzero $a \in \mathcal{A}$ there exists $t \geq 0$ such that $\left\|Z_{t} i(a)\right\|_{\hat{\varphi}}<\|a\|_{\varphi}$.

(c) for some $t \geq 0$, the $n$-tuple $\left\{Q \widehat{T}_{t} i\left(e_{j}\right) \mid j=1,2, \cdots n\right\}$ is linearly independent, i.e., $Q \widehat{T}_{t} i(\mathcal{A})$ has dimension $n$.

(d) for some $\varepsilon \geq 0, t \geq 0$, and all $x \in \mathcal{A}_{[0, \infty)}$,

$$
\left\|Z_{t} x\right\|_{\widehat{\varphi}} \leq(1-\varepsilon)\|x\|_{\widehat{\varphi}} .
$$

Proof. By Lemma 4.1. condition (a) obviously implies (b).

(b) $\Longrightarrow(\mathrm{c})$ : By the semigroup property of $Z_{t}$, the function $t \mapsto\left\|Z_{t} i(a)\right\|_{\hat{\varphi}}$ is decreasing for all $a \in \mathcal{A}$, in particular for all $a$ in the unit sphere of $\mathcal{A}$. And since the unit sphere is compact, in condition (b) $t$ can be chosen uniformly in $a$. 
Now, suppose that $\left\{Q \widehat{T}_{t} i\left(e_{j}\right) \mid j=1,2, \cdots, n\right\}$ is linearly dependent, i.e., for some $\lambda_{1}, \lambda_{2}, \cdots, \lambda_{n} \in \mathbb{C}:$

$$
\sum_{j=1}^{n} \lambda_{j} Q \widehat{T}_{t} i\left(e_{j}\right)=0 .
$$

Then we would have an $a \in \mathcal{A}$, namely $a:=\sum_{j=1}^{n} \lambda_{j} e_{j}$ for which $Q \widehat{T}_{t} i(a)=0$ so that

$$
\left\|Z_{t} i(a)\right\|_{\widehat{\varphi}}^{2}=1-\left\|Q \widehat{T}_{t} i(a)\right\|_{\widehat{\varphi}}^{2}=1=\|a\|_{\varphi}
$$

contradicting (b). This proves the linear independence in (c).

$(\mathrm{c}) \Longrightarrow(\mathrm{d})$ : We may assume that the vectors $e_{1}, \cdots, e_{n}$ form a $\varphi$-orthonormal basis of $(\mathcal{A}, \varphi)$, and since the coupling is of a tensor type, we may put $Q \widehat{T}_{t} i\left(e_{j}\right)=: \mathbf{1} \otimes g_{j}$ with $g_{j} \in \mathcal{C}_{[0, \infty)}$. Let $G$ denote the $n \times n$ matrix with entries $G_{i j}:=\psi\left(g_{i}^{*} g_{j}\right)$. Then $G$ is a positive matrix; let $\varepsilon$ denote its smallest eigenvalue. By the linear independence of the vectors $g_{1}, g_{2}, \cdots, g_{n}$, we have $\varepsilon>0$. Now consider $x \in \mathcal{A}_{[0, \infty)}$. Write

$$
x=\sum_{j=1}^{n} e_{j} \otimes f_{j}, \quad \text { with } \quad f_{j} \in \mathcal{C}_{[0, \infty)} .
$$

We calculate:

$$
\begin{aligned}
Q \widehat{T}_{t}(x) & =\sum_{j=1}^{n} Q\left(\widehat{T}_{t}\left(e_{j} \otimes \mathbf{1}\right) \widehat{T}_{t}\left(\mathbf{1} \otimes f_{j}\right)\right) \\
& =\sum_{j=1}^{n}\left(Q \widehat{T}_{t} i\left(e_{j}\right)\right) \cdot\left(\mathbf{1} \otimes S_{t} f_{j}\right) \\
& =\sum_{j=1}^{n} \mathbf{1} \otimes\left(g_{j} \cdot S_{t} f_{j}\right) .
\end{aligned}
$$

Now, since $g_{j} \in \mathcal{C}_{[0, t]}$ and $S_{t} f_{j} \in \mathcal{C}_{[t, \infty)}$, we find

$$
\begin{aligned}
\left\|Q \widehat{T}_{t}(x)\right\|_{\widehat{\varphi}}^{2} & =\left\|\sum_{j=1}^{n} g_{j} \cdot S_{t} f_{j}\right\|_{\psi}^{2} \\
& =\sum_{j=1}^{n} \sum_{k=1}^{n}\left\langle g_{j} \cdot S_{t} f_{j}, g_{k} \cdot S_{t} f_{k}\right\rangle_{\psi} \\
& =\sum_{j=1}^{n} \sum_{k=1}^{n}\left\langle g_{j}, g_{k}\right\rangle_{\psi}\left\langle f_{j}, f_{k}\right\rangle_{\psi} \\
& \geq \varepsilon \sum_{j=1}^{n}\left\|f_{j}\right\|_{\psi}^{2} \\
& =\varepsilon\|x\|_{\widehat{\varphi}}^{2} .
\end{aligned}
$$

So

$$
\left\|Z_{t}(x)\right\|_{\widehat{\varphi}}^{2} \leq\left\|Q^{\perp} \widehat{T}_{t}(x)\right\|_{\widehat{\varphi}}^{2} \leq(1-\varepsilon)\|x\|_{\widehat{\varphi}}^{2} .
$$


(d) $\Longrightarrow\left(\right.$ a): By the semigroup property of $Z_{t}$, we have

$$
\left\|Z_{n t} x\right\|_{\widehat{\varphi}}^{2} \leq(1-\varepsilon)^{n}\|x\|_{\widehat{\varphi}}^{2} .
$$

As $s \mapsto\left\|Z_{s} x\right\|_{\hat{\varphi}}$ is decreasing, it follows that $\lim _{t \rightarrow \infty}\left\|Z_{t} x\right\|_{\widehat{\varphi}}=0$ for all $x \in \mathcal{A}_{[0, \infty)}$, in particular for $x=i(a), a \in \mathcal{A}$. The assertion follows by Lemma 4.1.

\section{$\S 5$. Example: a classical Markov chain.}

Consider the stationary Markov chain with state space $X:=\{0,1,2\}$ and transition probability matrix

$$
T:=\left(\begin{array}{ccc}
\frac{1}{2} & \frac{1}{2} & 0 \\
\frac{1}{2} & 0 & \frac{1}{2} \\
0 & \frac{1}{2} & \frac{1}{2}
\end{array}\right) .
$$

This chain has the unique equilibrium distribution $\mu:=\left(\frac{1}{3}, \frac{1}{3}, \frac{1}{3}\right)$.

We shall represent this Markov chain by coupling $(X, \mu)$ to a Bernoulli shift. Let $(Y, \nu)$ denote the probability space $\left(\{0,1\},\left(\frac{1}{2}, \frac{1}{2}\right)\right)$, and let a coupling between $(X, \mu)$ and $(Y, \nu)^{\mathbb{Z}}$ be effected by the permutation $\gamma: X \times Y \rightarrow X \times Y$ described by the diagram

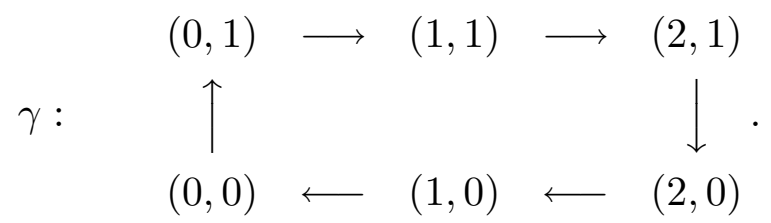

We shall denote the components of $\gamma$ as follows:

$$
\gamma(\xi, \eta)=\left(\gamma_{X}(\xi, \eta), \gamma_{Y}(\xi, \eta)\right) \in X \times Y, \quad(\xi \in X=\{0,1,2\}, \eta \in Y=\{0,1\}) .
$$

Clearly $\gamma$ preserves the product measure $\mu \otimes \nu$ on $X \times Y$. Now define

$$
\begin{aligned}
& \Omega:=X \times Y^{\mathbb{Z}}, \\
& \widehat{\mu}:=\mu \otimes\left(\bigotimes_{\mathbb{Z}} \nu\right) .
\end{aligned}
$$

We shall refer to the elements of $\Omega$ using the following suggestive notation:

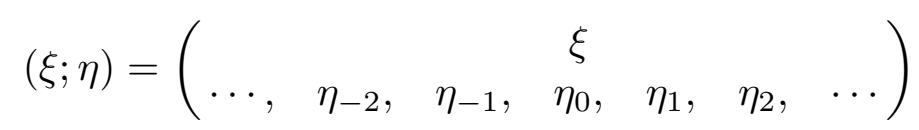

We define the transformation $\widehat{\gamma}$ and the shift $\sigma$ by

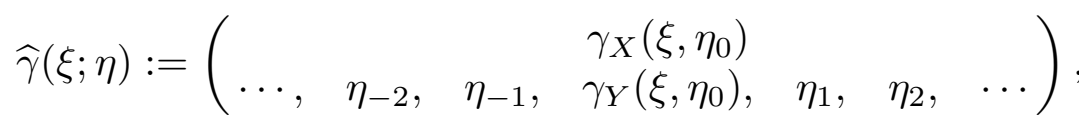

$$
\begin{aligned}
& \sigma(\xi ; \eta):=\left(\begin{array}{cccccc}
\xi & & & \\
\ldots, & \eta_{-1}, & \eta_{0}, & \eta_{1}, & \eta_{2}, & \eta_{3},
\end{array}\right) . \\
& -10 \text { - }
\end{aligned}
$$


Put

$$
\widehat{\tau}:=\sigma \circ \widehat{\gamma} .
$$

In the language of Section 1 we now have a stationary Markov process with transition operators $T^{n},(n \in \mathbb{N})$, on the space $(\mathcal{A}, \varphi)$ given by

$$
\mathcal{A}=\mathbb{C}^{3}, \quad \varphi(f):=\frac{1}{3}(f(0)+f(1)+f(2))=\int_{X} f d \mu .
$$

Indeed, put $\widehat{\mathcal{A}}:=L^{\infty}(\Omega, \widehat{\mu})$ and $\widehat{\varphi}(g):=\int_{\Omega} g d \widehat{\mu},(g \in \widehat{\mathcal{A}})$. Let

$$
\begin{aligned}
i: \mathcal{A} \rightarrow \widehat{\mathcal{A}}: & i(f)(\xi ; \eta)=f(\xi) ; \\
P: \widehat{\mathcal{A}} \rightarrow \mathcal{A}: & (P g)(\xi):=\int_{Y} \mathbb{Z}^{g(\xi ; \eta) \nu} \nu^{\mathbb{Z}}(d \eta) ; \\
\widehat{T}: \widehat{\mathcal{A}} \rightarrow \widehat{\mathcal{A}}: & \widehat{T}(g):=g \circ \widehat{\tau} .
\end{aligned}
$$

Then by an easy calculation one finds that

$$
(P \circ \widehat{T} \circ i)(f)(\xi)=(T f)(\xi),
$$

where $T$ is viewed as a linear map $\mathbb{C}^{3} \rightarrow \mathbb{C}^{3}$.

We shall show that this coupling representation is asymptotically complete, by applying our criterion of Theorem $4.2(\mathrm{c})$.

Take $t=2$ in Theorem $4.2(\mathrm{c})$, and let $e_{j}, j=0,1,2$, denote the canonical basis of $\mathcal{A}=\mathbb{C}^{3}$. We calculate the vectors $h_{j}:=Q \circ \widehat{T}^{2} \circ i\left(e_{j}\right) \in \mathcal{C}=L^{\infty}\left(Y^{\mathbb{Z}}, \nu^{\mathbb{Z}}\right)$ :

$$
\begin{aligned}
h_{j}(\eta) & =Q \circ \widehat{T}^{2} \circ i\left(e_{j}\right)(\eta) \\
& =\int_{X} i\left(e_{j}\right)\left(\widehat{\tau}^{2}(\xi ; \eta)\right) \mu(d \xi) \\
& =\int_{X} i\left(e_{j}\right)(\widehat{\gamma} \circ \sigma \circ \widehat{\gamma}(\xi ; \eta)) \mu(d \xi) \\
& =\frac{1}{3} \#\left\{\xi \in X \mid \gamma_{X}\left(\gamma_{X}\left(\xi, \eta_{0}\right), \eta_{1}\right)=j\right\} .
\end{aligned}
$$

Since the $h_{j}(\eta),(j=0,1,2)$ depend only on $\eta_{0}$ and $\eta_{1}$, we can express them as vectors in $\mathbb{C}^{4}$ :

$$
h_{0}=\frac{1}{3}(3,1,0,0), \quad h_{1}=\frac{1}{3}(0,2,2,0), \quad h_{2}=\frac{1}{3}(0,0,1,3) .
$$

Clearly, these vectors are linearly independent. Hence by Theorem 4.2 the coupling representation is asymptotically complete.

Next we shall calculate the scattering operator $\Phi_{-}$. Writing $\gamma_{n}$ for the map which applies $\gamma$ to $X \times\left(\right.$ the $n$-th component of $\left.Y^{\mathbb{Z}}\right)$, we may write

$$
\begin{aligned}
\Phi_{-} g= & \lim _{n \rightarrow \infty} S^{-n} \circ \widehat{T}^{n} g \\
= & \lim _{n \rightarrow \infty} g \circ(\sigma \widehat{\gamma})^{n} \circ \sigma^{-n} \\
= & \lim _{n \rightarrow \infty} g \circ \gamma_{-1} \circ \gamma_{-2} \circ \cdots \circ \gamma_{-n} . \\
& \quad 11-
\end{aligned}
$$


Now let $x_{0} \in \widehat{\mathcal{A}}$ be the function $(\xi ; \eta) \mapsto \xi$ and let $y_{k}:(\xi ; \eta) \mapsto \eta_{k}$. Note that $x_{0}$ and $y_{k},(k \in \mathbb{Z})$ generate $\widehat{\mathcal{A}}$. Furthermore, let bin $(\eta)$ for $\eta \in\{0,1\}^{\mathbb{Z}}$ denote the number whose binary expansion is given by the negative part of $\eta$ :

$$
\operatorname{bin}(\eta):=\sum_{k=1}^{\infty} \eta_{-k} 2^{-k}
$$

Let $[r]$ denote the integer part of the real number $r$.

Proposition 5.1. The scattering operator $\Phi_{-}$on an element of $\widehat{\mathcal{A}}$ is determined almost everywhere by

$$
\begin{gathered}
\Phi_{-}\left(x_{0}\right)(\eta)=[3 \operatorname{bin}(\eta)]= \begin{cases}0, & \text { if } \operatorname{bin}(\eta) \in\left[0, \frac{1}{3}\right) ; \\
1, & \text { if } \operatorname{bin}(\eta) \in\left(\frac{1}{3}, \frac{2}{3}\right) ; \\
2, & \text { if } \operatorname{bin}(\eta) \in\left(\frac{2}{3}, 1\right] .\end{cases} \\
\Phi_{-}\left(y_{k}\right)=y_{k} \quad \text { for } k \geq 0, \text { and for } k<0: \\
\Phi_{-}\left(y_{k}\right)(\eta)= \begin{cases}0, & \text { if }\left(2^{k} \operatorname{bin}(\eta) \bmod 1\right) \in\left[0, \frac{1}{3}\right) ; \\
\eta_{k}, & \text { if }\left(2^{k} \operatorname{bin}(\eta) \bmod 1\right) \in\left(\frac{1}{3}, \frac{2}{3}\right) ; \\
1, & \text { if }\left(2^{k} \operatorname{bin}(\eta) \bmod 1\right) \in\left(\frac{2}{3}, 1\right] .\end{cases}
\end{gathered}
$$

Proof. First note that $S^{-n} \circ \widehat{T}^{n}\left(y_{k}\right)=y_{k}$ for all $n, k \geq 0$, so that $\Phi\left(y_{k}\right)=y_{k}$. Second, for all $n \geq 0$ we have

$$
\begin{aligned}
S^{-n} \circ \widehat{T}^{n}\left(x_{0}\right)(\xi ; \eta) & =x_{0} \circ \gamma_{-1} \circ \gamma_{-2} \circ \cdots \circ \gamma_{-n}(\xi ; \eta) \\
& =\gamma_{X}\left(\gamma_{X}\left(\cdots\left(\gamma_{X}\left(\xi ; \eta_{-n}\right), \eta_{-n+1} \cdots\right), \eta_{-2}\right), \eta_{-1}\right) .
\end{aligned}
$$

The right hand side becomes constant as a function of $\xi$ and of $n$ as soon as two neighbouring components of $\left(\eta_{-n}, \eta_{-n+1}, \cdots, \eta_{-2}, \eta_{-1}\right)$ are equal, since for all $\xi \in\{0,1,2\}:$

$$
\gamma_{X}\left(\gamma_{X}(\xi, 0), 0\right)=0 \quad \text { and } \quad \gamma_{X}\left(\gamma_{X}(\xi, 1), 1\right)=2
$$

In particular, if $n \geq M(\eta):=\min \left\{m \geq 0 \mid \eta_{-m}=\eta_{-m-1}\right\}$ :

$$
x_{0}\left(\gamma_{-1} \circ \gamma_{-2} \circ \cdots \circ \gamma_{-n}(\xi ; \eta)\right)=x_{0}\left(\gamma_{-1} \circ \gamma_{-2} \circ \cdots \circ \gamma_{-M(\eta)+1}(\widetilde{\xi}, \eta)\right) \text {, }
$$

where

$$
\widetilde{\xi}:= \begin{cases}0, & \text { if } \eta_{-M(\eta)}=0 \\ 2, & \text { if } \eta_{-M(\eta)}=1 .\end{cases}
$$

Since $\widehat{\mu}[M(\eta)<\infty]=1$, it follows that eventually $S^{-n} \circ \widehat{T}^{n}\left(x_{0}\right)(\xi ; \eta)$ becomes independent of $n$ and of $\xi$ with probability 1. The convergence in $L^{2}(\Omega, \widehat{\mu})$ follows by the dominated convergence theorem. Finally, since $\eta_{-1}, \eta_{-2}, \cdots, \eta_{-M(\eta)}$ is an alternating sequence of 0 's and 1's, the value of the limit is for almost all $\eta$ given by

$$
\Phi_{-}\left(x_{0}\right)(\eta)= \begin{cases}0, & \text { if } \eta_{-1}=0 \text { and } \eta_{-M(\eta)}=0, \\ 1, & \text { if } \eta_{-1}=0 \text { and } \eta_{-M(\eta)}=1, \\ 1, & \text { if } \eta_{-1}=1 \text { and } \eta_{-M(\eta)}=0, \\ 2, & \text { if } \eta_{-1}=1 \text { and } \eta_{-M(\eta)}=1\end{cases}
$$

This is equal to the value of $\Phi_{-}\left(x_{0}\right)(\eta)$ claimed in the theorem. Third, by a similar argument one proves the expression for $\Phi_{-}\left(y_{k}\right)$. 
We have now obtained a translation representation $\left(Y^{\mathbb{Z}}, \nu^{\mathbb{Z}}, S ; j=\Phi_{-}\right)$of the full Markov chain in the noise algebra, as one can check explicitly.

\section{$\S 6 . \quad$ Asymptotic completeness in quantum stochastic calculus.}

As another application of Theorem 4.2 we consider the coupling of a finite dimensional matrix algebra to Bose noise. This is quite a satisfactory physical model for an atom or molecule in the electromagnetic field, provided that the widths of its spectral lines are small compared with the frequencies of the radiation the particle is exposed to. In [RoM] the result of the present paper was applied to this model, which was used to calculate the nontrivial physical phenomenon known as the 'dynamical Stark effect', namely the splitting of a fluorescence line into three parts with specified height and width ratios, when the atom is subjected to extremely strong, almost resonant radiation. The results agreed with those in the physics literature, both theoretical [Mol] and experimental [SSH]. A different result on the existence of scattering operators in the context of quantum stochastic calculus is found in $[\mathrm{FaS}]$.

The model mentioned above falls in the class of continuous time Markov processes over a finite state space driven by Bose noise, as described briefly below. In this section, we cast the criterion Theorem 4.2(c) for asymptotic completeness into a manageable form for these Markov processes.

For $\mathcal{A}$ we take the algebra $M_{n}$ of all complex $n \times n$ matrices, on which a faithful state $\varphi$ is given by

$$
\varphi(x):=\operatorname{tr}(\rho x) .
$$

Here $\rho$ is a diagonal matrix with strictly positive diagonal elements, summing up to 1 . The modular group of $(\mathcal{A}, \varphi)$ is given by

$$
\sigma_{t}(x):=\rho^{-i t} x \rho^{i t} \text {. }
$$

The eigenvalues of the operator $\sigma_{t}$ are of the form $e^{ \pm i t \beta_{j}}$, for some positive numbers $\beta_{j}, 1 \leq j \leq m$. We shall couple the system $(\mathcal{A}, \varphi)$ to Bose noise (Cf. [Par], [LiM]). Let $\mathcal{C}$ denote the Weyl algebra over an $m$-fold direct sum $\bigoplus_{m} L^{2}(\mathbb{R})$. It is linearly generated by the Weyl operators, on which the state $\psi$ is given by

$$
\psi\left(W\left(f_{1} \oplus f_{2} \oplus \cdots \oplus f_{m}\right)\right):=\exp \left(-\frac{1}{2} \sum_{j=1}^{m} \operatorname{coth}\left(\frac{1}{2} \beta_{j}\right)\left\|f_{j}\right\|^{2}\right) .
$$

A time evolution $S_{t}$ on $\mathcal{C}$ is induced by the right shift on the functions $f_{1}, f_{2}, \cdots, f_{m} \in$ $L^{2}(\mathbb{R})$.

In physical terms the dynamical system $\left(\mathcal{C}, \psi, S_{t}\right)$ describes a noise source consisting of $m$ channels which contain thermal radiation at inverse temperatures $\beta_{1}, \beta_{2}, \cdots, \beta_{m}$.

On the GNS representation of $(\mathcal{C}, \psi)$ we have canonical annihilation operators $A_{j}(t),(j=1, \cdots, m)$, (cf. [Par]). 
In $[\mathrm{LiM}]$, Section 9, Markov processes $\left(\widehat{\mathcal{A}}, \widehat{\varphi}, \widehat{T}_{t} ; i\right)$ over $(\mathcal{A}, \varphi)$ are constructed by coupling to these Bose noise channels. They are of the following form.

$$
\begin{array}{rlrl}
\widehat{\mathcal{A}} & :=\mathcal{A} \otimes \mathcal{C} & \text { with } i(a):=a \otimes \mathbf{1} ; \\
\widehat{\varphi} & :=\varphi \otimes \psi & \text { with } P(x \otimes y):=\psi(y) x ; & \\
\widehat{T}_{t}(a) & :=u_{t}^{*}\left(\operatorname{id} \otimes S_{t}\right)(a) u_{t}, \quad(t \geq 0) ; \quad \widehat{T}_{t}:=\left(\widehat{T}_{-t}\right)^{-1}, \quad(t<0),
\end{array}
$$

Here $u_{t}$ is the solution of the quantum stochastic differential equation $d u_{t}=$

$$
\left(\sum_{j=1}^{m}\left(v_{j} \otimes d A_{j}^{*}(t)-v_{j}^{*} \otimes d A_{j}(t)-\frac{1}{2}\left(c_{j}^{+} v_{j}^{*} v_{j}+c_{j}^{-} v_{j} v_{j}^{*}\right) \otimes \mathbf{1} \cdot d t\right)+(i h \otimes \mathbf{1}) \cdot d t\right) u_{t},
$$

with initial condition $u_{0}=\mathbf{1}$, where

$$
c_{j}^{+}:=\sqrt{\frac{e^{\beta_{j}}}{e^{\beta_{j}}+1}}, \quad c_{j}^{-}:=\sqrt{\frac{1}{e^{\beta_{j}}+1}},
$$

and $v_{j} \in \mathcal{A}=M_{n}$ are eigenvectors of the modular group $\sigma_{t}$ of $(\mathcal{A}, \varphi)$ and $h$ is fixed under $\sigma_{t}$.

The semigroup $T_{t}=e^{-t L}$ of transition operators on $(\mathcal{A}, \varphi)$ associated to this Markov process is obtained as $T_{t}:=P \circ \widehat{T}_{t} \circ i$, and has the infinitesimal generator $L: \mathcal{A} \rightarrow \mathcal{A}$ given by

$$
L(a)=i[h, a]-\frac{1}{2} \sum_{j=1}^{m}\left(c_{j}^{+}\left(v_{j}^{*} v_{j} a-2 v_{j}^{*} a v_{j}+a v_{j}^{*} v_{j}\right)+c_{j}^{-}\left(v_{j} v_{j}^{*} a-2 v_{j} a v_{j}^{*}+a v_{j} v_{j}^{*}\right)\right) .
$$

Now, the key observation in $[\mathrm{LiM}]$ and $[\mathrm{RoM}]$ which we need here is the following. Let $v_{j}^{+1}:=v_{j}$ and $v_{j}^{-1}:=v_{j}^{*}$. Let $L_{j}^{ \pm 1}$ be the operator $x \mapsto\left[v_{j}^{ \pm 1}, x\right]$ on $\mathcal{A}$.

Observation 6.1. If $Q$ is the projection onto the future noise algebra $\mathcal{C}_{[0, \infty)}$, then

$$
\begin{aligned}
& \left\|Q \widehat{T}_{t}(x \otimes \mathbf{1})\right\|^{2}= \\
& \sum_{k=0}^{\infty} \sum_{j \in\{1, \cdots, m\}^{k}} \sum_{\varepsilon \in\{-1,+1\}^{k}} c_{j(1)}^{\varepsilon(1)} \cdots c_{j(k)}^{\varepsilon(k)} \\
& \int_{0 \leq s_{1} \leq \cdots \leq s_{k} \leq t}\left|\varphi\left(T_{t-s_{k}} L_{j(k)}^{\varepsilon(k)} T_{s_{k}-s_{k-1}} \cdots T_{s_{2}-s_{1}} L_{j(1)}^{\varepsilon(1)} T_{s_{1}}(x)\right)\right|^{2} d s_{1} \cdots d s_{k} .
\end{aligned}
$$

Together with Theorem 4.2 this leads to the following results concerning asymptotic completeness.

Proposition 6.2. The system $\left(\widehat{\mathcal{A}}, \widehat{\varphi}, \widehat{T}_{t} ; i\right)$ described above is asymptotically complete if and only if for all nonzero $x \in M_{n}$ there are $t>0, k \in \mathbb{N}$, and $s_{1}, s_{2}, \cdots, s_{k}$ satisfying $0 \leq s_{1} \leq \cdots \leq s_{k} \leq t, j(1), \cdots, j(k) \in\{1, \cdots m\}$ and $\varepsilon \in\{-1,1\}^{m}$ such that

$$
\varphi\left(T_{t-s_{k}} L_{j(k)}^{\varepsilon(k)} \cdots T_{s_{2}-s_{1}} L_{j(1)}^{\varepsilon(1)} T_{s_{1}}(x)\right) \neq 0 .
$$


In particular, if $\varphi$ is a trace, i.e. $\rho=\frac{1}{n} \mathbf{1}$ in the above, then $\varphi \circ T_{t}=\varphi$ and $\varphi \circ L_{j}^{\varepsilon}=0$, so that the system can never be asymptotically complete for $n \geq 2$. This agrees with the general idea that a trace state $\varphi$ should correspond to noise at infinite temperature, i.e. classical noise [KüMa]. Obviously, if $\mathcal{C}$ is commutative there can be no isomorphism $j$ between $\mathcal{C}$ and $\mathcal{C} \otimes M_{n}$.

Proof. The proposition follows immediately from Theorem 4.2(b), Observation 6.1 and the continuity in $s_{1}, s_{2}, \cdots, s_{k}$ of the integrand therein.

Corollary 6.3. A sufficient condition for $\left(\widehat{\mathcal{A}}, \widehat{\varphi}, \widehat{T}_{t} ; i\right)$ to be asymptotically complete is that for all $x \in M_{n}$ there exists $k \in \mathbb{N}, j \in\{1,2, \cdots, m\}^{k}$, and $\varepsilon \in$ $\{-1,+1\}^{k}$ such that

$$
\varphi\left(L_{j(k)}^{\varepsilon(k)} \cdots L_{j(1)}^{\varepsilon(1)}(x)\right) \neq 0
$$

In particular, the Wigner-Weisskopf atom as treated in [RoM], which is the case $n=2, m=1$,

$$
v=v_{1}=\left(\begin{array}{cc}
0 & 0 \\
1 & 0
\end{array}\right) ; \quad \rho=\frac{1}{e^{\beta}+1}\left(\begin{array}{cc}
e^{\beta} & 0 \\
0 & 1
\end{array}\right)
$$

is asymptotically complete.

\section{References}

[Acc] L. Accardi (1978). On the quantum Feynman-Kac formula. Rend. Sem. Mat. Fis. Milano 48, 135-179.

[AFL] L. Accardi, A. Frigerio, J.T. Lewis (1982). Quantum stochastic processes. Publications of the R.I.M.S., Kyoto, 18, 97-133.

[Bia] P. Biane (1995). Calcul stochastique non-commutatif. In: Lectures on Probability Theory, Ecole d'Eté de Probabilités de Saint-Flour XXIII-1993 (P. Bernard ed.) Lecture Notes in Mathematics 1608 Springer, Berlin 1995, 1-96.

[BKS] M. Bożejko, B. Kümmerer, R. Speicher (1997). q-Gaussian processes: noncommutative and classical aspects. Commun. Math. Phys. 185, 155-175.

[BSp] M. Bożejko, R. Speicher (1991). An example of a generalized Brownian motion I. Commun. Math. Phys. 137, 519-531.

[FaS] F. Fagnola, K. Sinha (1993). In: Festschrift Volume in Honour of G. Kallianpur (Cambanis et al. eds.) Springer 1993.

[FrO] N. Friedman, D.S. Ornstein (1971). An isomorphism of weak Bernoulli transformations. Advances in Math. 5, 365-394.

[KeS] M. Keane, M. Smorodinsky (1979). Finitary isomorphisms of irredicible Markov shifts. Israel Journal of Mathematics 34 281-286.

[Küm1] B. Kümmerer (1985). Markov dilations on $W^{*}$-algebras. Journ. Funct. Anal. 63, $139-177$.

[Küm2] B. Kümmerer (1985). On the structure of Markov dilations on $W^{*}$-algebras. Quantum Probability and Applications II (L. Accardi, W. von Waldenfels, eds.) Springer, Berlin 1985, 318-331. 
[Küm3] B. Kümmerer (1988). Survey on a theory of non-commutative stationary Markov processes. Quantum Probability and Applications III (L. Accardi, W. von Waldenfels, ed.) Springer, Berlin 1988, 154-182.

[Küm4] B. Kümmerer (1994). Stochastic processes with values in $M_{n}$ as coupling to free evolutions. Preprint.

[KüMa] B. Kümmerer, H. Maassen (1987). The essentially commutative dilations of dynamical semigroups on $M_{n}$. Commun. Math. Phys. 109, 1-22.

[KüS] B. Kümmerer, W. Schröder (1985). A new construction of unitary dilations: singular coupling to white noise. Quantum Probability and Applications II (L. Accardi, W. von Waldenfels, eds.) Springer, Berlin 1985, 332-347.

[LiM] J.M. Lindsay, H. Maassen (1992). Stochastic calculus for quantum Brownian motion of non-minimal variance. In: Mark Kac seminar on probability and physics, Syllabus 1987-1992. CWI Syllabus 32, Amsterdam, 97-167.

[LPh] P.D. Lax, R.S. Phillips (1967). Scattering theory. Academic Press, New York.

[Mey] P.A. Meyer (1993). Quantum Probability for Probabilists. Lecture Notes in Mathematics 1538 Springer, Berlin 1993.

[Mol] B.R. Mollow (1969). Power spectrum of light scattered by two-level systems. Phys. Rev. 188, 1969-1975.

[Par] K.R. Parthasarathy (1992). An introduction to quantum stochastic calculus. Birkhäuser, Basel-Boston-Berlin.

[Rob] D.W. Robinson (1973). Return to equilibrium. Commun. Math. Phys. 31, 171-189.

[RoM] P. Robinson, H. Maassen (1991). Quantum stochastic calculus and the dynamical Stark effect. Reports Math. Phys. 30, 185-203.

[SSH] F. Schuda, C.R. Stroud, M. Hercher (1974). Observation of resonant Stark effect at optical frequencies. Journ. Phys. B7, 198.

[Voi 1] D. Voiculescu (1985). Symmetries of some reduced free product $\mathrm{C}^{*}$-algebras. In: Operator algebras and their connection with topology and ergodic theory. Springer Lecture Notes in Mathematics 1132, 556-588.

[Voi 2] D. Voiculescu (1986). Addition of certain non-commuting random variables. J. Funct. Anal. 66,323-346.

[WaM] D. Walls, G.J. Milburn (1995). Quantum Optics. Springer, New York 1995. 